\title{
Neurocysticercosis in Epileptic Patients Seen in the Emergency Department of the Municipality of Piedade, Sao Paulo
}

\author{
Jose Augusto Camargo a, b, Reinaldo Jose Gianini ${ }^{\mathrm{a}}$, Moises Goldbaum ${ }^{\mathrm{a}}$
}

\begin{abstract}
Background: Neurocysticercosis (NCC) is a parasitic disease that affects the central nervous system. Its main clinical manifestation is seizures. The objective of this study was to investigate the prevalence of NCC in epileptic patients seen in the Emergency Department of the Municipality of Piedade, Sao Paulo.
\end{abstract}

Methods: A total of 136 patients with a history of seizures seen in the Municipal Emergency Department of Piedade/SP were evaluated.

Results: There were 24 confirmed cases of NCC among the 136 patients with seizures, corresponding to a prevalence of $17.6 \%$.

Conclusion: An important prevalence of NCC was observed in the sample studied. Measures to raise the awareness of the population about the disease and preventive strategies should be adopted by the competent bodies.

Keywords: Neurocysticercosis; Seizures

\section{Introduction}

Neurocysticercosis (NCC) is a term used to define involvement of the central nervous system (CNS) by larval forms of the tapeworm Taenia solium. This disease is the most common cause of epilepsy in adults worldwide and one of the parasitic infections most frequently associated with chronic morbidity in all continents, including North America. Clinical manifestations other than seizures are dementia, intracranial hypertension, and meningitis. A number of studies suggest that, in its vesicular form, the cysticercus can persist for many years in the CNS, with the patient remaining asymptomatic $[1,2]$.

Despite its importance, the worldwide morbidity due to NCC is poorly appreciated. Financial resources for research

Manuscript accepted for publication January 24, 2017

aFaculdade de Medicina, Universidade de Sao Paulo, Sao Paulo, SP, Brazil ${ }^{b}$ Corresponding Author: Jose Augusto Camargo, Faculdade de Medicina, Universidade de Sao Paulo, Sao Paulo, SP, Brazil. Email: jacneuro@hotmail.com

doi: https://doi.org/10.14740/jnr418e are scarce, a fact preventing investigators from making the necessary advances in the prevention and treatment of this neuroinfection and thus preventing millions of cases of individuals with epilepsy. It is estimated that 50 million people worldwide have NCC, with 50,000 annual deaths [3].

Brazil is among the countries strongly affected by the taeniasis-cysticercosis complex. In most states and their municipalities, precarious hygiene habits, diets and basic sanitation conditions, among other factors, contribute to the occurrence of this disease. The State of Sao Paulo, the richest state in Brazil, faces the same problem in all of its regions, regardless of their socioeconomic situation. Among the few studies on this topic conducted in Brazil, one demonstrated an incidence of 74.1 cases of NCC per 100,000 inhabitants in Ribeirao Preto, northeastern region of the state $[1,4]$.

Within this context, the objective of this study, at this time, was to investigate strictly the current situation in the Municipality of Piedade, Sao Paulo State, Brazil, evaluating the prevalence of NCC among epileptic citizens seen in the Municipal Emergency Department.

\section{Materials and Methods}

\section{Patients}

A descriptive study was conducted on 136 patients of both genders with a history of seizures at least once in their life. The patients were referred by physicians of the Municipal Emergency Department for neurological assessment by the responsible researcher.

NCC was diagnosed according to the criteria of Del Bruto et al (2001) [5].

\section{Study area and period}

Piedade (Sao Paulo)

\section{1) History}

Piedade has been known as the onion capital and used to be the largest producer in Brazil. Today, the agriculture of the municipality is diverse, producing fruits and vegetables, especially artichoke, strawberries and onions. 


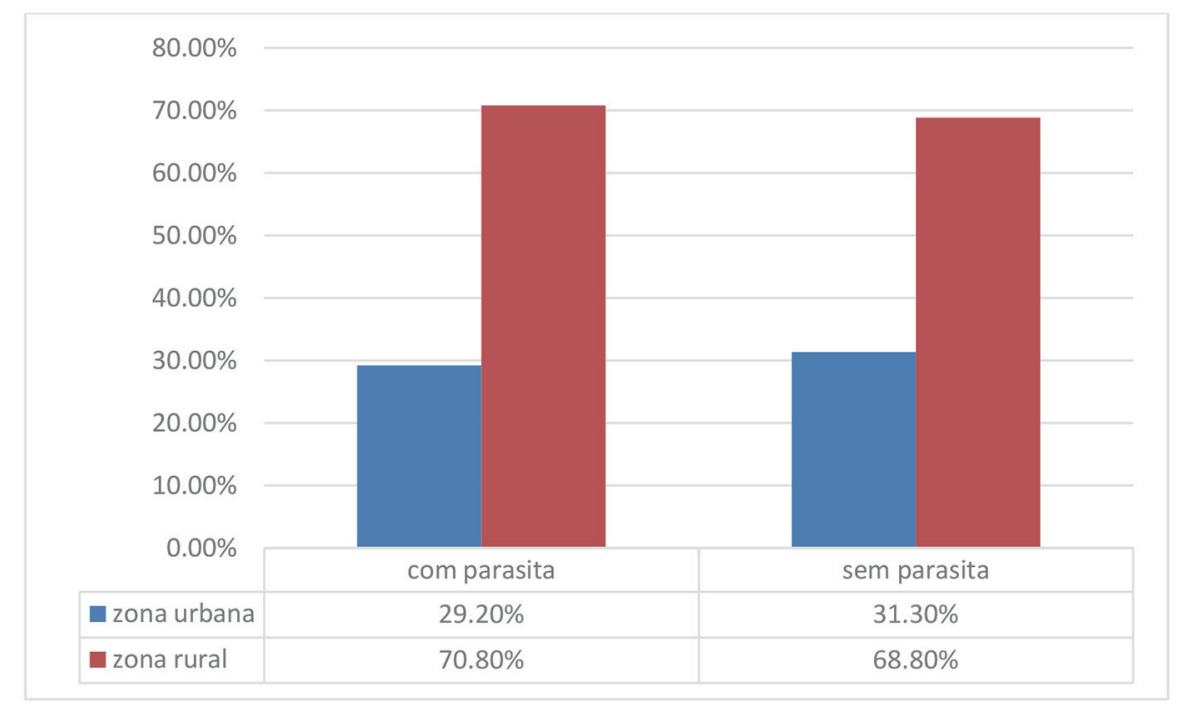

Figure 1. Percentage of patients living in the urban and rural areas with and without parasites.

\section{2) Geography}

The municipality is located between mountains, in the inland of the Serra do Mar, in an ecological preservation area. Several rivers, streams and creeks belonging to the basins of the Tiete, Paranapanema and Ribeira de Iguape rivers cross Piedade. The municipality comprises an area of $745.52 \mathrm{~km}^{2}$.

\section{3) Demography}

The population descended from pioneers and natives and has not received significant slave labor. Foreign immigration only occurred in the mid-20th century, with the arrival of Japanese and Italians. In view of its very cold and humid climate and because of its reserves, the town has received numerous German, Swiss and Austrian immigrants, but also Dutch, Belgian, Danish, Swedish and Norwegian immigrants, and does therefore not present the formation of relevant colonies.

The total population is 52,214 inhabitants; of these, 23,782 live in the urban area and 28,432 in the rural area. There are 26,602 men and 25,612 women (source: CENSO 2010/IBGE).

\section{Study period}

The study was conducted from May 2014 to August 2015 and included all residents of the urban and rural areas of the Municipality of Piedade/SP.

\section{Data collection}

A questionnaire was developed according to the objectives of the study. The data were collected by personal interview using the structured and pretested questionnaire after the sub- jects had signed the free informed consent form for voluntary participation. The data were collected exclusively by the responsible researcher. The participants were informed about the objective of the study and confidentiality and were identified by their initials and last name.

Subjects less than 12 years of age and pregnant women were excluded.

Computed tomography (CT) scans and/or cranial magnetic resonance imaging (MRI) were available for all cases studied.

\section{Ethical approval}

The study was approved by the Ethics Committee of the University of Sao Paulo School of Medicine (2013/1068-FMUSP).

\section{Statistical analysis}

The data were analyzed using the STATA 10.0 software. Analysis consisted of description of the distribution of absolute and relative $(\%)$ frequencies of all variables studied. The association between the presence of NCC and the other variables was evaluated using Fisher's exact test or Chi-squared test, adopting a level of significance of $<0.05$ [6].

\section{Results}

The questionnaire was applied to 136 patients, including 54 (39.71\%) males and $82(60.29 \%)$ females ranging in age from 13 to 86 years. In the case of subjects younger than 18 years, the questions were answered by the parents or legal representative. Among the participants with parasites detected by the imaging methods, $29.2 \%$ lived in the urban area and $70.8 \%$ in the rural area (Fig. 1). 


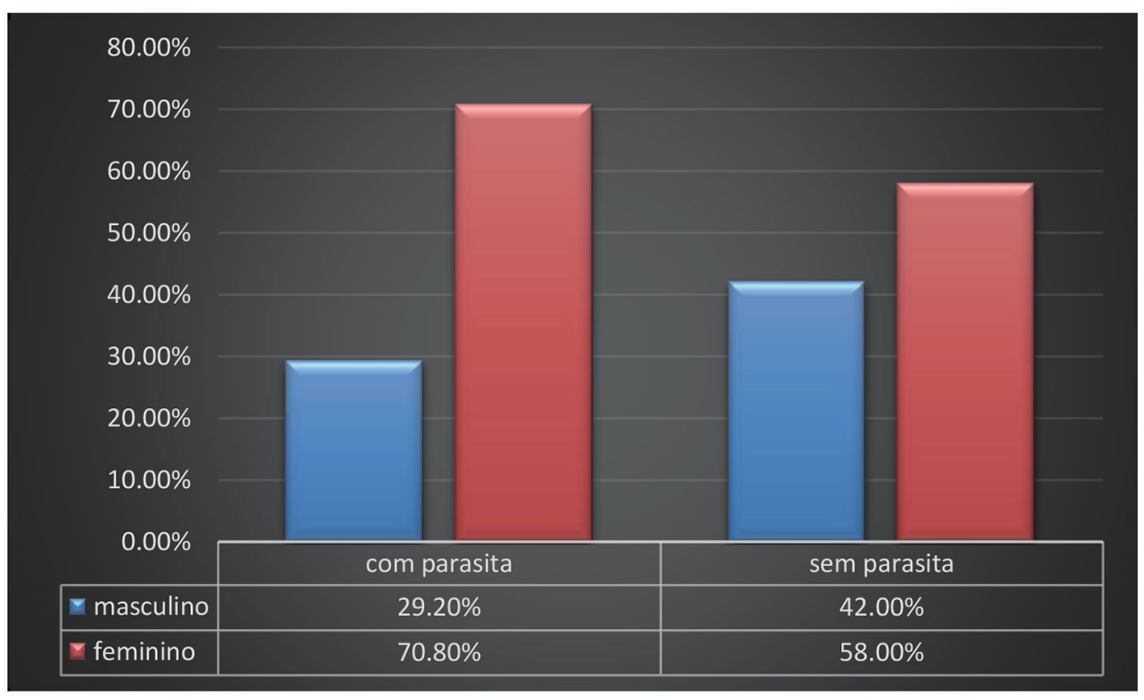

Figure 2. Percentage of male and female patients with and without parasites.

Only calcified parasites were found in $100 \%$ of contaminated cases. The percentage of patients with parasites was 29.2\% among males and 70.8\% among females (Fig. 2).

All patients had a history of seizures, with at least one episode in their life, and were submitted to CT and/or cranial MRI.

All cases were informed about their test results, prescriptions were given of medicines in case of need and oriented to continue the treatment with the specialist.

The results were analyzed and reviewed by the principal investigator along with two other researchers.

\section{Discussion}

The prevalence of NCC in Brazil is unknown due to the lack of mandatory notification in most states and to the inaccessibility of the majority of the population to diagnostic tests of the disease. The clinical findings are variable and the polymorphic manifestations depend not only on the location, but also on the number of parasites, the developmental stage of the cysticerci (viable or calcified), and host characteristics. The cerebral calcifications observed in the present study represent a serious health problem since they can cause irreversible changes in the patient and may require treatment and medication use throughout life $[7,8]$. The diagnosis of NCC is made by a combination of immunological methods and neuroimaging techniques [9-12]. In the area studied here, other individuals may by contaminated and asymptomatic, carrying different forms of the parasite that can be present in the CNS for many years without causing exuberant clinical symptoms [1,2]. Unfortunately, many health services are ineffective in notifying cases, a fact that renders geographic mapping deficient and, consequently, impairs eradication of the disease. The factors that contribute to the endemic nature of the taeniasis/cysticercosis complex are multiple and complex and are closely related to personal hygiene habits and family and environmental factors. In this respect, it is necessary to raise the awareness of government agencies regarding broad investment in collective health programs and to attribute to health education the importance that it deserves in the control of the taeniasis/cysticercosis complex, considering the severe consequences of this disease or collective health and the lack of access of the majority of the population to diagnostic methods.

An effective control program of the disease should include strict inspection methods of pork quality and guidance of the population regarding the need to consume well-cooked pork and pork products, considering that the parasite can lodge in any part of the animal's body. The animal or its products should be purchased in reliable establishments submitted to health inspections by the competent bodies. Vegetables and fruits must be very well washed with filtered or boiled water before consumption. The water used for human consumption must also be filtered or boiled. Guidance regarding handwashing before eating, when preparing food and after using the toilet is imperative. In view of the biological cycle of the parasite, the adequate disposal of human feces through septic tanks and cesspools would be a major step towards controlling the disease. Raising awareness of the population and mobilizing resources by the competent bodies for research and control of cysticercosis are urgently needed not only in the area studied, but around the world.

\section{Conflicts of Interest}

The authors have declared no conflicts of interest.

\section{References}

1. Takayanagui OM, Leite JP. Neurocisticercose. Rev Soc Bras Med Trop. 2001;34:283-290.

2. Grewal JS, Kaur S, Bhatti G, Sawhney IM, Ganguly NK, Mahajan RC, Malla N. Cellular immune responses in hu- 
man neurocysticercosis. Parasitol Res. 2000;86(6):500503.

3. Engels D, Urbani C, Belotto A, Meslin F, Savioli L. The control of human (neuro) cysticercosis: which way forward? Acta Trop. 2003;87(1):177-182.

4. Agapejev S. Epidemiology of neurocysticercosis in Brazil. Rev Inst Med Trop Sao Paulo. 1996;38(3):207-216.

5. Del Brutto OH, Rajshekhar V, White AC, Jr., Tsang VC, Nash TE, Takayanagui OM, Schantz PM, et al. Proposed diagnostic criteria for neurocysticercosis. Neurology. 2001;57(2):177-183.

6. Fisher RA (1934) Statistical Methods for Research Workers. 5th Edition, Edinburgh: Oliver and Boyd.

7. Camargo JA, Bertolucci PH. Quantification of Amino Acid Neurotransmitters in Cerebrospinal Fluid of Patients with Neurocysticercosis. Open Neurol J. 2015;9:15-20.
8. Camargo JA, Bertolucci PH. Quantification of Fas protein in CSF of patients with neurocysticercosis. Arq Neuropsiquiatr. 2012;70(4):262-266.

9. Garcia HH, Evans CA, Nash TE, Takayanagui OM, White AC, Jr., Botero D, Rajshekhar V, et al. Current consensus guidelines for treatment of neurocysticercosis. Clin Microbiol Rev. 2002;15(4):747-756.

10. Ostrosky-Zeichner L, Estanol B. Immunopathogenesis of neurocysticercosis: is damage mediated by the host immune response? Int J Parasitol. 1999;29(4):649-650.

11. White AC, Jr. Neurocysticercosis: updates on epidemiology, pathogenesis, diagnosis, and management. Annu Rev Med. 2000;51:187-206.

12. Carpio A, Romo ML. State of the art in neurocysticercosis: Imaging and epidemiology. Asian Pac J Trop Med. 2016;9(8):821-822. 